\title{
Physical inactivity is associated with decreased growth differentiation factor II in chronic obstructive pulmonary disease
}

This article was published in the following Dove Press journal: International Journal of COPD

\section{Rie Tanaka' \\ Hisatoshi Sugiura' \\ Mitsuhiro Yamada' \\ Tomohiro Ichikawa' \\ Akira Koarai' \\ Naoya Fujino' \\ Satoru Yanagisawa' \\ Katsuhiro Onodera' \\ Tadahisa Numakura' \\ Kei Sato' \\ Yorihiko Kyogoku' \\ Hirohito Sano' \\ Shun Yamanaka' \\ Tatsuma Okazaki' \\ Tsutomu Tamada' \\ Motohiko Miura ${ }^{2}$ \\ Tsuneyuki Takahashi ${ }^{3}$ \\ Masakazu Ichinose' \\ 'Department of Respiratory Medicine, \\ Tohoku University Graduate \\ School of Medicine, Aoba-ku, \\ Sendai, Japan; ${ }^{2}$ Department of Respiratory Medicine, Tohoku Rosai Hospital, Aoba-ku, Sendai, Japan; ${ }^{3}$ Department of Internal Medicine, Tohoku Medical and Pharmaceutical University Wakabayashi Hospital, Wakabayashi-ku, Sendai, Japan}

Correspondence: Hisatoshi Sugiura Department of Respiratory Medicine, Tohoku University Graduate School of Medicine, I-I Seiryo-machi, Aoba-ku, Sendai 980-8574, Japan

Tel +8I $227 \mid 78539$

Fax +8I 227178549

Email sugiura@rm.med.tohoku.ac.jp
Background: Growth differentiation factor 11 (GDF11) is reported to possess anti-aging and rejuvenating effects, including muscle regeneration and to be highly expressed in skeletal muscle. Recently, we demonstrated that the levels of plasma GDF11 were decreased in COPD. However, the effect of decreased circulating GDF11 in the pathophysiology of COPD remains unknown. The aim of this study is to investigate the association between the plasma GDF11 levels and various clinical parameters in patients with COPD.

Patients and methods: Eighteen ex-smokers as control subjects and 70 COPD patients participated in the current study. We measured the levels of plasma GDF11 using immunoblotting, lung function, physical activity using a triaxial accelerometer, quadriceps strength, exercise capacity, and systemic inflammatory markers. We investigated the association between the levels of plasma GDF11 and these clinical parameters.

Results: The levels of plasma GDF11 in the COPD patients had significant positive correlations with the data of lung function. Furthermore, the levels of plasma GDF11 were significantly correlated with the physical activity, quadriceps strength, and exercise capacity. Moreover, the levels of plasma GDF11 were significantly correlated with the data of inflammatory markers. Although various factors were related to GDF11, the multiple regression analysis showed that physical activity was significantly associated with the levels of plasma GDF11.

Conclusion: Physical inactivity was significantly related to the decreased GDF11 levels in COPD, which might be useful for understanding the pathogenesis of COPD. Clarifying the relationships between the physical inactivity and GDF11 may reveal a potentially attractive therapeutic approach in COPD via increasing the plasma levels of GDF11.

Keywords: physical activity, muscle strength, rejuvenating factor, COPD

\section{Introduction}

There is increasing evidence that senescence plays a crucial role in the pathogenesis of COPD. ${ }^{1-4}$ In fact, cellular senescence in various resident cells is accelerated in COPD lungs. ${ }^{5-7}$ Senescence of lung resident cells is reported to cause destruction of the normal lung architecture ${ }^{2,3,5}$ and is believed to be associated with the systemic inflammation observed in COPD. ${ }^{8-10}$ In this context, the acceleration of lung cellular senescence could be involved in the development and progress of the disease.

Growth differentiation factor 11 (GDF11) is a member of the transforming growth factor beta superfamily. ${ }^{11}$ GDF11 has been focused on as an anti-aging and rejuvenating factor. ${ }^{12-14}$ In particular, Sinha et al showed that treatment with GDF11 regenerated the injured muscle and restored satellite cell function, resulting in increased muscle strength and exercise endurance in aged mice. ${ }^{14}$ The expression of GDF11 was 
observed in various organs of mice and highly present in skeletal muscle. ${ }^{12}$

Recently, we demonstrated that the production of GDF11 in lung fibroblasts and airway epithelial cells from patients with COPD was decreased compared with those from control subjects. ${ }^{15}$ We showed that treatment with GDF11 inhibited the cigarette smoke extract (CSE)-induced cellular senescence and inflammation in lung resident cells. ${ }^{15}$ We also demonstrated that the administration of GDF11 suppressed the elastase-induced cellular senescence and enlargement of alveolar spaces in vivo, ${ }^{15}$ suggesting that GDF11 might be involved in the pathogenesis of COPD. Furthermore, we found that the amounts of plasma GDF11 in patients with COPD were decreased compared with those in control subjects. ${ }^{15}$ However, it remains unknown whether there is an association between the decrease in plasma GDF11 and various clinical data related to the pathogenesis of COPD.

In the current study, we aimed to investigate the correlation between the levels of plasma GDF11 and various clinical parameters, including lung function, physical activity, quadriceps strength, exercise capacity, and systemic inflammatory markers in patients with COPD.

\section{Materials and methods Study subjects}

Seventy outpatients with COPD and 18 age-matched healthy subjects were recruited into the current study from Tohoku University Hospital, Tohoku Rosai Hospital, and Tohoku Medical and Pharmaceutical University Wakabayashi Hospital. The study subjects were enrolled from separate cohorts of a previous study. ${ }^{15}$ All patients with COPD were aged $>40$ years, had a former smoking history of $>10$ pack-years, and were diagnosed according to the Global Initiative for Chronic Obstructive Lung Disease (GOLD) documents. ${ }^{16}$ Age-matched control subjects with a smoking history of $>10$ pack-years and normal lung function were enrolled. All patients with COPD and control subjects had been free of infections of the respiratory tract and exacerbations due to any reasons for at least 2 months prior to the study. All participants had quit smoking for at least 1 year. Patients with COPD and control subjects with pathologic conditions, including cerebrovascular diseases, rheumatoid arthritis, and arteriosclerosis obliterans, which affected the physical activity, were excluded from the study. Written informed consent was obtained from all subjects. All experiments in the current study were approved by the ethics committee of Tohoku University Graduate School of Medicine (approval number: \#2013-1-074), Tohoku Rosai Hospital (approval number: \#15-11), and Tohoku Medical and Pharmaceutical University Wakabayashi Hospital (approval number: \#2015-1-024).

\section{Study design}

Lung function tests were performed using CHESTAC-8800 (Chest Ltd., Tokyo, Japan) and whole blood was collected into vacutainer tubes containing an anticoagulant. The plasma samples were obtained by centrifugation of the tubes at 3,000 rpm for 10 minutes and then stored at $-80^{\circ} \mathrm{C}$ until the assay. Physical activity was measured by a triaxial accelerometer for 2 weeks and the levels of physical activity were analyzed. Then, the exercise capacity was measured by means of 6-minute walking tests, after which the quadriceps strength was measured.

\section{Measurement of plasma GDFI I levels}

The levels of plasma GDF11 were assessed by immunoblotting. ${ }^{15}$ The plasma samples were diluted with saline and the diluted samples were mixed with sample buffer (Bio-Rad Laboratories, Hercules, CA, USA). Equal amounts of protein were loaded and separated by electrophoresis on $10 \%$ sodium dodecyl sulfate polyacrylamide gels. After electrophoresis, the separated proteins were transferred to a polyvinylidene difluoride membrane (Merck Millipore Ltd., Darmstadt, Germany). We used a different antibody raised against GDF11 specifically that does not recognize myostatin (1:1,000 dilution, R\&D Systems Inc., Minneapolis, MN, USA). ${ }^{15}$ Bound antibodies were visualized using a peroxidase-conjugated anti-mouse goat antibody (1:2,000 dilution, Santa Cruz Biotechnology Inc., Dallas, TX, USA) and enhanced chemiluminescence (GE Healthcare Ltd., Buckinghamshire, UK) with a chemiluminescence imaging system (LAS-4000 mini; Fujifilm CO., Tokyo, Japan). Ponceau S staining was used to evaluate the amounts of protein. The GDF11 levels were calculated by measuring the intensity of the bands. Band intensity was quantified using ImageJ $1.48 \mathrm{v}$ software (National Institutes of Health, Bethesda, MD, USA).

\section{Assessment of physical activity}

Physical activity was measured by a triaxial accelerometer (Actimarker; Panasonic CO., Osaka, Japan), which can continuously monitor activity for over 1 month, as previously reported. ${ }^{17,18}$ Briefly, patients wore the accelerometer during the daytime for 14 consecutive days. From among the 14 days monitoring data, except the first and last days, the data of 5 non-rainy weekdays were analyzed according to previous studies. ${ }^{17-19}$ We measured the parameters of physical activity as follows: number of steps, duration of activity at $\geq 2.0, \geq 2.5$, and $\geq 3.0$ metabolic equivalents (METs). 


\section{Measurement of quadriceps strength}

Isometric quadriceps maximum voluntary contraction (QMVC) was assessed using an Biodex System3 Dynamometer (Biodex Medical Systems, Shirley, NY, USA). The subjects were seated and their hip and knee joints were flexed at 90 degrees. The subjects performed isometric contraction for 3 seconds, the maximum force was measured, and predicted QMVC was calculated as described in a previous report. ${ }^{20}$

\section{Measurements of 6-minute walking distance (6MWD)}

The 6MWD was measured according to American Thoracic Society guidelines. ${ }^{21}$

\section{Measurement of plasma levels of interleukin-6 (IL-6), high sensitivity C-reactive protein (hs-CRP), fibrinogen, and malondialdehyde (MDA)}

Plasma IL- 6 and hs-CRP were measured by a commercially available enzyme-linked immunosorbent assay kit (R\&D Systems Inc.), according to the manufacturer's instructions. Fibrinogen was measured using an automatic blood coagulation analyzer (CS-5100; Sysmex CO., Kobe, Japan). MDA, which is a marker of lipid peroxidation, was measured as thiobarbituric acid reactive substances according to the manufacturer's instructions (Abcam plc., Cambridge, UK).

\section{Statistical analysis}

When we designed this study, we consulted with a statistician in our institution and he estimated how many subjects were needed in this study. Based on our statistician's opinion, we calculated the appropriate sample sizes using the values of $\alpha=0.05$, effect size $=0.3$, and $1-\beta=0.7$. Accordingly, at least 67 patients were needed. In the current study, we included 70 patients in the study.

The data are expressed as mean \pm SD or median (interquartile range) as appropriate. Comparisons of the number of steps, duration of activity, and 6MWD between the groups were analyzed with Kruskal-Wallis test followed by Dunn's test. The remaining data were statistically analyzed with oneway analysis of variance followed by Tukey's test. Statistical correlation analyses were performed using Spearman's test. A linear regression analysis was performed using the method of least squares. Multivariable models were used to determine associations between GDF11 and other variables. Multiple regression analysis was performed using GDF11 as the dependent variable. The independent variables were selected using a stepwise approach and included in the model (ie, number of steps and QMVC). Potential confounders, such as lung function, exercise capacity, and inflammatory markers were also included in the model if they showed statistical significance $(p<0.05)$. Among the parameters of lung function, forced vital capacity (FVC) \% predicted, forced expiratory volume in 1 second $\left(\mathrm{FEV}_{1}\right) \%$ predicted, inspiratory capacity (IC), and diffusing capacity of the lung for carbon monoxide $\left(\mathrm{D}_{\mathrm{Lco}}{ }^{\prime}\right) \%$ predicted showed statistical significance by univariate analysis. To avoid multicollinearity, we selected $\mathrm{FEV}_{1} \%$ predicted in this model. Smoking history (pack-years) was included in the model and was independent of statistical significance. Statistical analysis was performed using GraphPad Prism 6 (GraphPad Software Inc., San Diego, CA, USA) and JMP v12 Pro (SAS Institute Inc., Cary, NC, USA). $p$-values $<0.05$ were considered significant.

\section{Results}

\section{Relationship between GDFII levels and lung function}

Eighteen control subjects and 70 patients with COPD (GOLD stage I/II, $n=37$; GOLD stage III/IV, $n=33$ ) took part in the current study. The characteristics of the study subjects are listed in Table 1. Compatible with the previous study, ${ }^{15}$

Table I Characteristics of the study subjects

\begin{tabular}{|c|c|c|c|}
\hline & \multirow[t]{2}{*}{ Control } & \multicolumn{2}{|l|}{ COPD } \\
\hline & & GOLD I/II & GOLD III/IV \\
\hline Subjects, $n$ & 18 & 37 & 33 \\
\hline Age, years & $69.2 \pm 6.2$ & $70.1 \pm 5.5$ & $71.2 \pm 6.2$ \\
\hline Males/females & $|7 /|$ & $36 / 1$ & $33 / 0$ \\
\hline $\begin{array}{l}\text { Smoking, } \\
\text { pack-years }\end{array}$ & $30.5 \pm 11.4$ & $60.0 \pm 27.5^{*}$ & $61.3 \pm 30.5^{*}$ \\
\hline $\mathrm{BMI}, \mathrm{kg} / \mathrm{m}^{2}$ & $24.0 \pm 3.2$ & $23.6 \pm 3.2$ & $21.0 \pm 3.5^{*, \neq}$ \\
\hline $\begin{array}{l}\text { mMRC dyspnea } \\
\text { score }\end{array}$ & $0.1 \pm 0.2$ & $0.9 \pm 0.8^{*}$ & $1.9 \pm 1.0^{*, \pm}$ \\
\hline FVC, \% predicted & $108.3 \pm 12.7$ & $99.5 \pm 16.0$ & $73.6 \pm 19.0^{* \neq \neq}$ \\
\hline $\mathrm{FEV}_{1}, \%$ predicted & $103.0 \pm 13.1$ & $70.8 \pm 12.2^{*}$ & $32.6 \pm 10.8^{* \neq \neq}$ \\
\hline $\mathrm{FEV}_{1} / \mathrm{FVC}, \%$ & $77.5 \pm 4.4$ & $58.1 \pm 9.0 *$ & $35.7 \pm 8.7^{*, \neq}$ \\
\hline IC, L & $2.8 \pm 0.5$ & $2.3 \pm 0.4^{*}$ & $1.8 \pm 0.5^{*, \neq}$ \\
\hline $\mathrm{D}_{\text {Lco }}$, \% predicted & I I $2.3 \pm 27 . \mid$ & $70.0 \pm 25.4^{*}$ & $43.2 \pm 22.6$ *., \\
\hline 6MWD, m & $510(470-558)$ & $450(400-484)^{*}$ & $350(208-402)^{* \neq+}$ \\
\hline LABA, n & - & 23 & 29 \\
\hline LAMA, n & - & 27 & 33 \\
\hline ICS, n & - & 8 & II \\
\hline
\end{tabular}

Notes: Data are presented as mean \pm SD or median (IQR). $*_{p}<0.01$ versus control, ${ }^{\ddagger} p<0.0$ I versus GOLD I/II.

Abbreviations: BMI, body mass index; $D_{\text {Lco }}$, diffusing capacity of the lung for carbon monoxide; FEV , forced expiratory volume in I second; FVC, forced vita capacity; GOLD, Global Initiative for Chronic Obstructive Lung Disease; IC, inspiratory capacity; ICS, inhaled corticosteroids; IQR, interquartile range; LABA, long-acting $\beta_{2}$ agonist; LAMA, long-acting muscarinic antagonist; mMRC, modified Medical Research Council; 6MWD, 6-minute walking distance. 
the levels of plasma GDF11 measured by immunoblotting were significantly decreased in the patients with COPD compared with those in the control subjects (Figure S1). We investigated the association between the plasma GDF11 levels and lung function. The levels of plasma GDF11 in the COPD patients had significantly positive correlations with the values of $\mathrm{FEV}_{1} \%$ predicted $(r=0.48, p<0.001$, Figure 1A), FVC $\%$ predicted ( $r=0.39, p=0.001$, Figure $1 \mathrm{~B})$, IC $(r=0.41, p<0.001$, Figure $1 \mathrm{C})$, and $\mathrm{D}_{\mathrm{Lco}} \%$ predicted $(r=0.36, p=0.003$, Figure 1D).

\section{Relationship between GDFI I levels and physical activity}

The physical activity measured by a triaxial accelerometer and the data of physical activity given in daily number of steps and intensity of physical activity (METs) are shown in Table 2. All data of the physical activity were significantly lower in the GOLD stage III/IV patients compared with those in the control subjects and GOLD stage I/II patients (Table 2). The levels of plasma GDF11 were significantly correlated with the number of steps $(r=0.65, p<0.001$,
Figure 2A), duration of $\geq 2.0$ METs $(r=0.57, p<0.001$, Figure 2B), $\geq 2.5 \operatorname{METs}(r=0.59, p<0.001$, Figure 2C), and $\geq 3.0$ METs $(r=0.53, p<0.001$, Figure 2D).

\section{Relationship between GDFII levels and muscle strength and exercise capacity}

Because GDF11 is reported to be highly expressed in skeletal muscle, ${ }^{12}$ we investigated the relationship between the GDF11 levels and muscle strength. The levels of plasma GDF11 had a significantly positive correlation with the values of QMVC \% predicted in the COPD patients $(r=0.37$, $p=0.009$, Figure 3A). Since exercise capacity is related to quadriceps strength in $\mathrm{COPD},{ }^{22,23}$ we further investigated correlations between the GDF11 levels and the 6MWD. The levels of plasma GDF11 had a significantly positive correlation with the 6MWD in the COPD patients $(r=0.43$, $p<0.001$, Figure 3B).

\section{GDFII and inflammatory markers}

Since cellular senescence is reported to be associated with systemic inflammation in COPD,${ }^{8-10}$ and GDF 11 , anti-aging
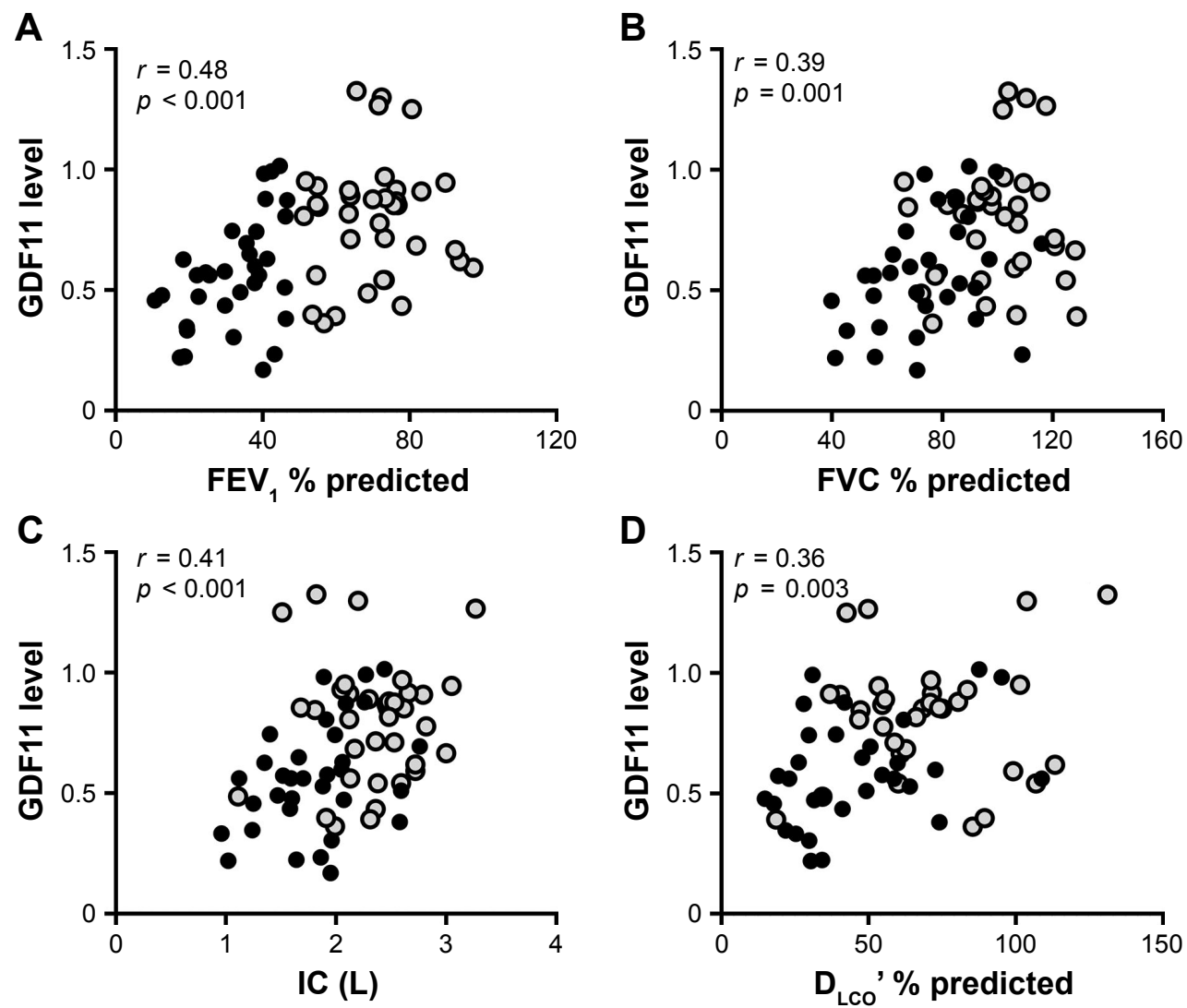

Figure I Correlations between the levels of GDFI I and lung function data.

Notes: Correlations between the levels of plasma GDFII and the values of FEV, \% predicted (A), FVC \% predicted (B), IC (C), and D $\mathrm{D}_{\text {Lco }} \%$ predicted (D) were investigated. Data were statistically analyzed by the Spearman's rank test. Closed circles (gray): GOLD stage I/II; closed circles (black): GOLD stage III/IV.

Abbreviations: $\mathrm{D}_{\text {Lco }}$ ', diffusing capacity of the lung for carbon monoxide; $F E V_{1}$, forced expiratory volume in I second; FVC, forced vital capacity; GDFI I, growth differentiation factor II; GOLD, Global Initiative for Chronic Obstructive Lung Disease; IC, inspiratory capacity. 
Table 2 Physical activity in the study subjects

\begin{tabular}{|c|c|c|c|}
\hline & \multirow[t]{2}{*}{ Control } & \multicolumn{2}{|l|}{ COPD } \\
\hline & & GOLD I/II & GOLD III/IV \\
\hline Subjects, $n$ & 18 & 37 & 33 \\
\hline Number of steps per day & $6,844(4,935-7,832)$ & $5,460(2,527-7,840)$ & $1,744(716-4,543)^{* *, \neq}$ \\
\hline Duration of $\geq 2.0 \mathrm{METs}, \mathrm{min} /$ day & $177(143-254)$ & $162(92-225)$ & $83(58-157)^{* \neq}$ \\
\hline Duration of $\geq 2.5 \mathrm{METs}, \mathrm{min} /$ day & $84(63-143)$ & 71 (35-95) & $27(11-62)^{* *, \ddagger}$ \\
\hline Duration of $\geq 3.0 \mathrm{METs}$, min/day & $37(25-5 I)$ & $25(12-43)$ & $8(3-19)^{* *, \neq}$ \\
\hline
\end{tabular}

Notes: Data are presented as median (IQR). ${ }^{*} p<0.0$ I, ${ }^{* *} p<0.001$ versus control, ${ }^{\star} p<0.0$ I versus GOLD I/II.

Abbreviations: GOLD, Global Initiative for Chronic Obstructive Lung Disease; METs, metabolic equivalents; IQR, interquartile range.

factor, reportedly possesses anti-inflammatory effects, ${ }^{15,24}$ we investigated the associations between the levels of plasma GDF11 and inflammatory markers, including IL-6, hs-CRP, fibrinogen, and MDA. We found that the levels of plasma GDF11 had negative correlations with the values of IL-6 $(r=-0.36, p=0.002$, Figure 4A) and hs-CRP $(r=-0.31$, $p=0.007$, Figure 4B), but not with those of fibrinogen $(r=-0.13, p=0.368$, Figure $4 \mathrm{C})$ or MDA $(r=-0.15$, $p=0.263$, Figure 4D).
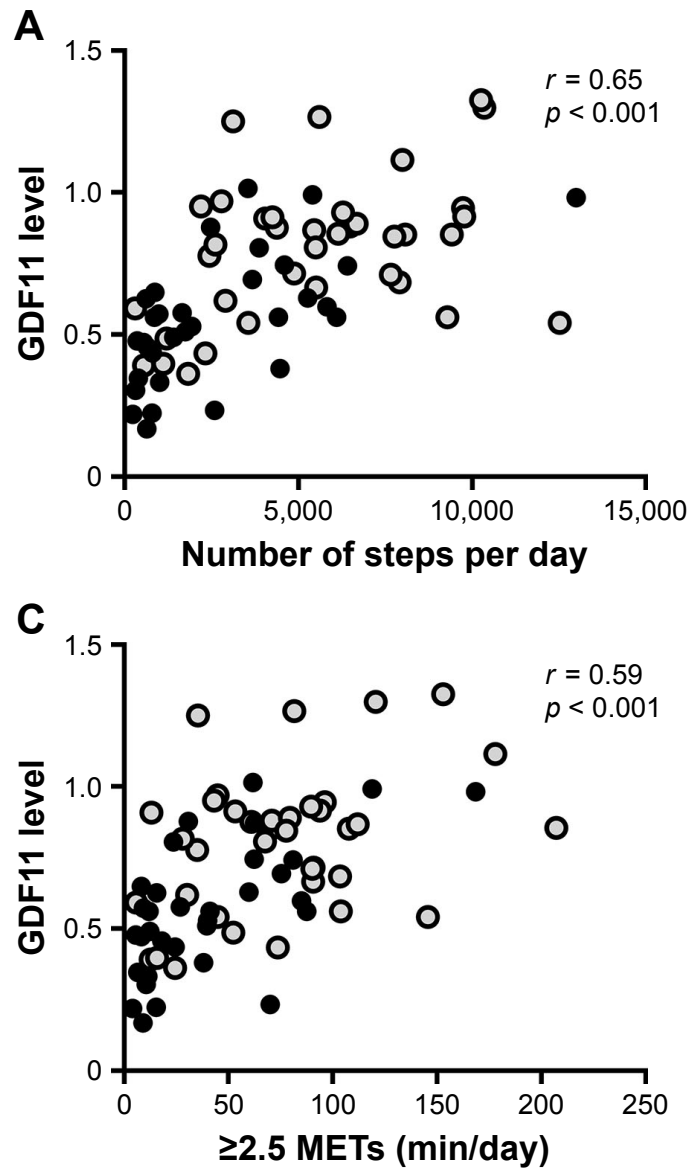

\section{Analysis of factors that influenced GDFI I}

We investigated the factors that influenced GDF11. We analyzed the factors related to the levels of plasma GDF11 by single linear regression analysis (Table 3 ). The data of lung function (FVC \% predicted, $\beta=0.64, p<0.001$; $\mathrm{FEV}_{1} \%$ predicted, $\beta=0.43, p<0.001$; IC, $\beta=0.66, p<0.001$; $\mathrm{D}_{\text {Lco }}, \%$ predicted, $\left.\beta=0.31, p=0.001\right), 6 \mathrm{MWD}(\beta=0.48$, $p<0.001)$, the number of steps $(\beta=0.28, p<0.001)$, the amounts of IL-6 $(\beta=-0.21, p=0.020)$, and QMVC \%
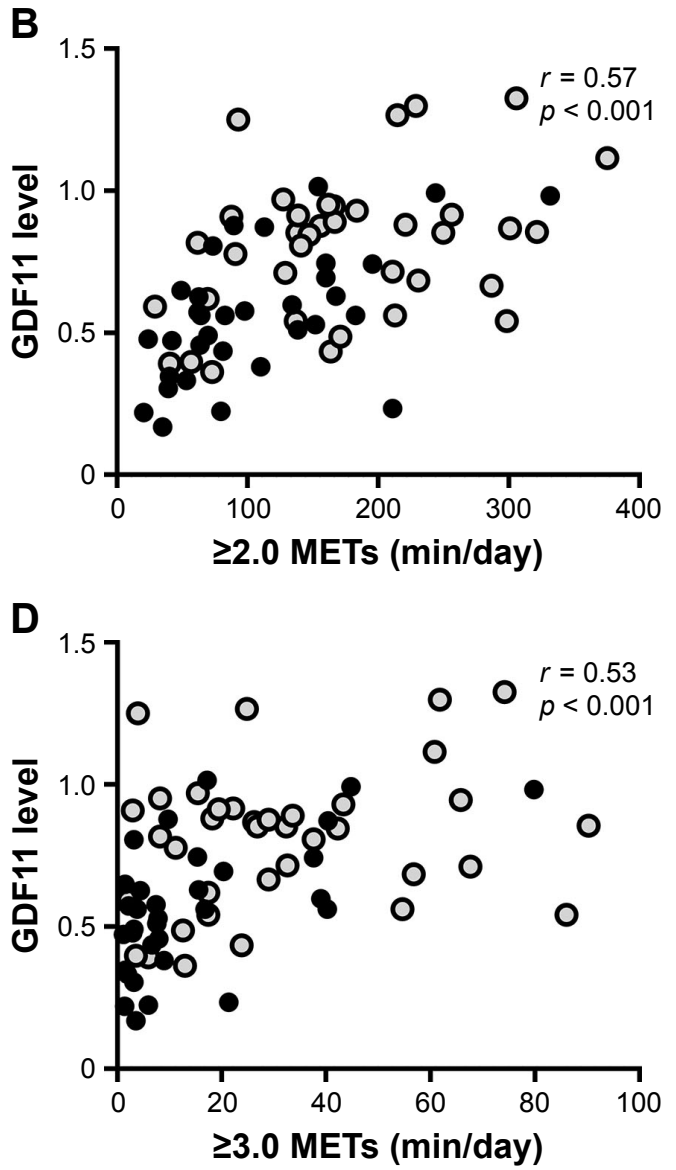

Figure 2 Correlations between the levels of GDFI I and physical activity.

Notes: Correlations between the levels of plasma GDFI I and parameters of physical activity including the number of steps (A), duration of $\geq 2.0$ METs (B), $\geq 2.5$ METs (C), and $\geq 3.0$ METs (D) were investigated. $r$ is the correlation coefficient. Data were statistically analyzed by the Spearman's rank test. Closed circles (gray): GOLD stage I/ll; closed circles (black): GOLD stage III/IV.

Abbreviations: GDFII, growth differentiation factor II; GOLD, Global Initiative for Chronic Obstructive Lung Disease; METs, metabolic equivalents. 

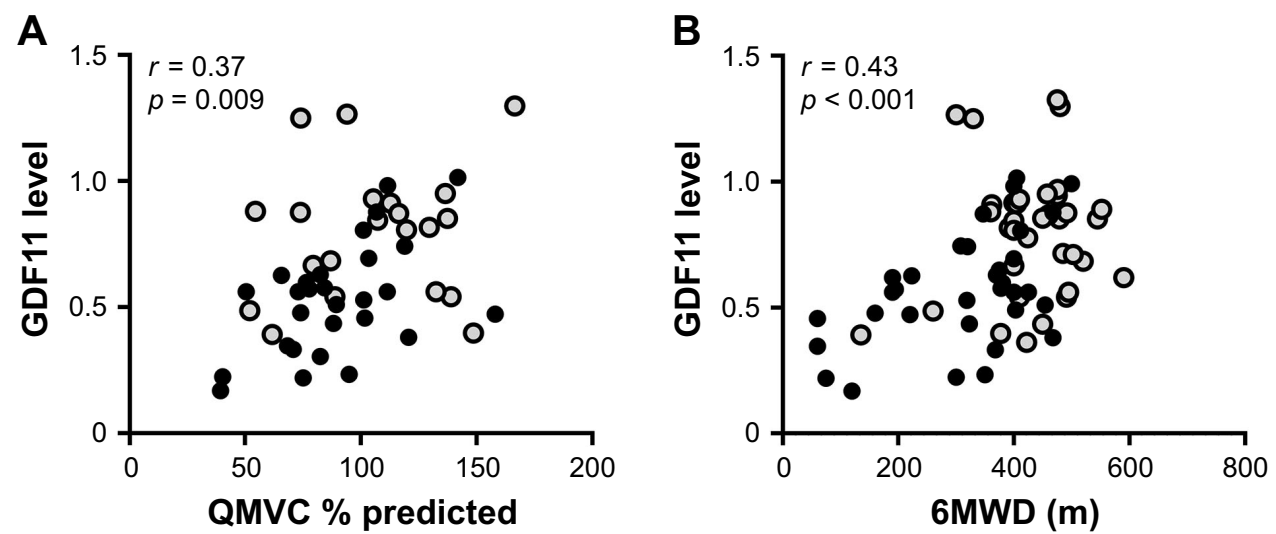

Figure 3 Correlations between the levels of GDFII and muscle strength and exercise capacity.

Notes: Correlations between the levels of plasma GDFII and the QMVC \% predicted (A) and the 6MWD (B) were investigated. $r$ is the correlation coefficient. Data were statistically analyzed by the Spearman's rank test. Closed circles (gray): GOLD stage I/Il; closed circles (black): GOLD stage III/IV.

Abbreviations: GDFII, growth differentiation factor II; GOLD, Global Initiative for Chronic Obstructive Lung Disease; 6MWD, 6-minute walking distance; QMVC, quadriceps maximal voluntary contraction.

predicted $(\beta=0.64, p<0.001)$ were significantly related to the levels of plasma GDF11 (Table 3). We further investigated the data by multiple regression analysis in the COPD group (Table 4). We found that the number of steps
( $\beta=0.20, p=0.045)$ was significantly related to the GDF11 (Table 4) independent of the lung function, suggesting that the physical activity might be a predictor of the levels of plasma GDF11 in COPD.
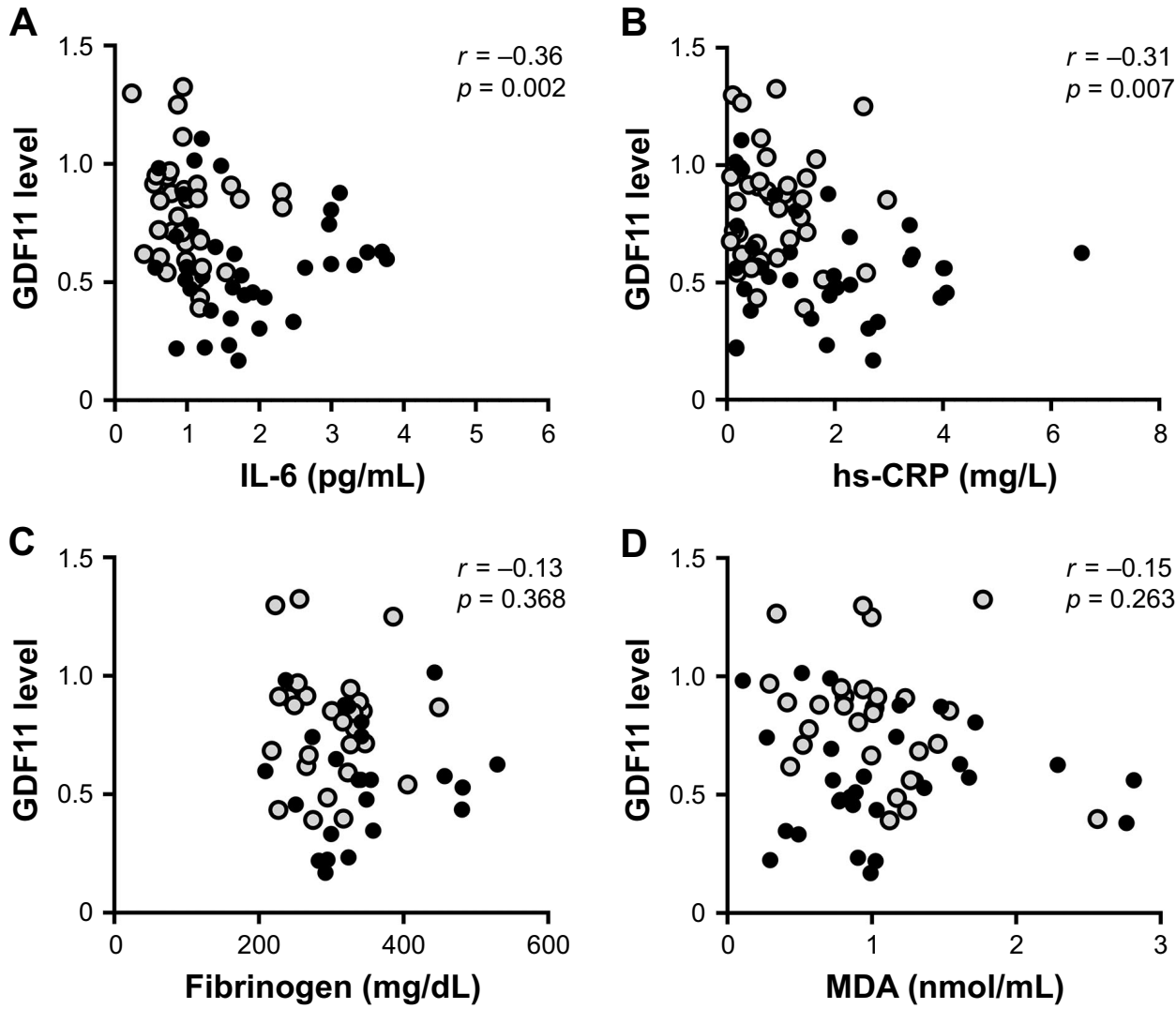

Figure 4 Correlations between the levels of GDFII and systemic inflammatory markers.

Notes: Correlations between the levels of plasma GDFII and the amounts of IL-6 (A), hs-CRP (B), fibrinogen (C), and MDA (D) were investigated. $r$ is the correlation coefficient. Data were analyzed by the Spearman's rank test. Closed circles (gray): GOLD stage I/II; closed circles (black): GOLD stage III/IV.

Abbreviations: GDFI I, growth differentiation factor I I; GOLD, Global Initiative for Chronic Obstructive Lung Disease; hs-CRP, high sensitivity C-reactive protein; IL-6, interleukin-6; MDA, malondialdehyde. 
Table 3 Single regression analysis of plasma GDFII in COPD patients

\begin{tabular}{lll}
\hline Variables & GDFI I & \\
\cline { 2 - 3 } & $\beta^{*}$ & p-value \\
\hline Age (years) & -0.33 & $0.6 I I$ \\
Smoking & -0.06 & 0.576 \\
FVC \% predicted & 0.64 & $<0.00$ I \\
FEV, \% predicted & 0.43 & $<0.00$ I \\
IC & 0.66 & $<0.00$ I \\
D ', \% predicted & 0.31 & 0.00 I \\
6MWD & 0.48 & $<0.00$ I \\
Number of steps & 0.28 & $<0.00$ I \\
IL-6 & $-0.2 I$ & 0.020 \\
QMVC \% predicted & 0.64 & $<0.00$ I \\
\hline
\end{tabular}

Notes: $\beta^{*}=$ regression coefficient. All data were log transformed. Data were analyzed by single linear regression analysis.

Abbreviations: $\mathrm{D}_{\text {Lco }}$, diffusing capacity of the lung for carbon monoxide; $F E V_{1}$, forced expiratory volume in I second; FVC, forced vital capacity; GDFI I, growth differentiation factor II; IC, inspiratory capacity; IL-6, interleukin-6; 6MWD, 6-minute walking distance; QMVC, quadriceps maximal voluntary contraction.

\section{Discussion}

The present study demonstrated that the levels of plasma GDF11, an anti-aging and rejuvenating factor, were significantly correlated with the lung function, levels of physical activity, quadriceps strength, exercise capacity, and systemic inflammatory markers. In addition, the multiple regression analysis showed that the number of steps was related to GDF11 in COPD. These results suggest that physical activity might be associated with the decreased plasma GDF11 in COPD.

In this study, we demonstrated that the levels of plasma GDF11 had significant correlations with the values of FEV $\%$ predicted and $\mathrm{D}_{\text {Lco }}, \%$ predicted, and were compatible with those of a previous study.$^{15}$ In addition, we showed correlations between the levels of plasma GDF11 and the values of FVC \% predicted and IC. These findings support that GDF11

Table 4 Multiple regression analysis of plasma GDFII in the COPD patients

\begin{tabular}{lll}
\hline Variables & GDFII \\
\cline { 2 - 3 } & $\beta^{*}$ & p-value \\
\hline Smoking & 0.23 & 0.078 \\
FEV $\%$ predicted & 0.05 & 0.735 \\
6MWD & 0.02 & 0.908 \\
Number of steps & 0.20 & 0.045 \\
IL-6 & -0.01 & 0.909 \\
QMVC \% predicted & 0.31 & 0.093 \\
\hline
\end{tabular}

Notes: $\beta^{*}=$ regression coefficient. All data were log transformed. Data were analyzed by multivariate linear regression. $R^{2}=0.50$ (adjusted $R^{2}=0.42$ ).

Abbreviations: $\mathrm{FEV}_{1}$, forced expiratory volume in I second; GDFII, growth differentiation factor II; IL-6, interleukin-6; 6MWD, 6-minute walking distance; QMVC, quadriceps maximal voluntary contraction. may be related to the pathogenesis of COPD. In our previous study, we demonstrated that the production of GDF11 in the lungs of the COPD patients was decreased compared with the control subjects. ${ }^{15}$ Moreover, treatment with GDF11 inhibited the CSE-induced cellular senescence in vitro. ${ }^{15}$ These findings suggested that GDF11 in lungs could possess an anti-senescent effect. However, the causes of the decrease in circulating GDF11 remain unclear, although the levels of plasma GDF11 in COPD patients were decreased compared with those in control subjects. ${ }^{15}$ One possible explanation is that the plasma GDF11 might spill over from the lungs into the blood stream because the expression of GDF11 in the lungs was decreased in COPD. Another possible explanation is that GDF11 is highly expressed in skeletal muscle, ${ }^{12}$ less so in lungs, suggesting that circulating GDF11 could be derived from skeletal muscles. In fact, many studies reported that limb muscle atrophy and weakness were observed in COPD patients. ${ }^{25,26}$ These findings prompted us to investigate the relationships between plasma GDF11 and clinical parameters, including physical activity. Actually, we found that the levels of plasma GDF11 had positive correlations with the quadriceps strength and physical activity in the present study. Furthermore, we demonstrated an association between the levels of plasma GDF11 and the exercise capacity. Exercise capacity was reported to be related to physical activity ${ }^{19,27}$ and quadriceps strength ${ }^{22,23}$ in COPD. Therefore, the levels of plasma GDF11 could be correlated with the 6MWD in the current study. In a previous study, the administration of GDF11 regenerated the injured muscle and restored the satellite cell function, resulting in increased muscle strength and exercise endurance in aged mice. ${ }^{14}$ Taken together, GDF11 might be a possible therapeutic approach for the muscle weakness of COPD. Further study is needed to reveal the effects of GDF11 on the muscle dysfunction in COPD.

Conversely, some myokines, including IL-6 and irisin were reportedly produced and released from contracting skeletal muscle into the circulation during exercise in healthy subjects. ${ }^{28-30}$ In addition, irisin was also upregulated after exercise in patients with COPD. ${ }^{31}$ However, it has not been elucidated whether the circulating GDF11 was increased by muscle contraction. Because GDF11 could increase muscle strength and endurance, it would be of interest to explore whether improvement of the physical activity could increase the production of circulating GDF11. To clarify this issue, we should investigate whether pulmonary rehabilitation or exercise training can improve the levels of circulating GDF11 in patients with COPD. 
In the current study, we demonstrated that the levels of plasma GDF11 had negative correlations with the amounts of IL-6 and hs-CRP. Previous reports showed that physical inactivity in patients with COPD could cause systemic inflammation. ${ }^{32,33}$ Therefore, the levels of plasma GDF11 might be correlated with the levels of inflammatory markers. Alternatively, GDF11 was reported to possess anti-inflammatory effects in vitro and in vivo studies. ${ }^{15,24}$ Thus, the decrease in GDF11 might be the cause of the systemic inflammation observed in COPD. No correlations were found between the levels of plasma GDF11 and the levels of MDA. MDA might be an insensitive marker of lipid peroxidation in the whole body, so the levels of MDA were not correlated with those of GDF11 in this study.

There are some limitations in this study. First, we could not measure the actual amounts of plasma GDF11 because they were semi-quantified by immunoblotting. We should establish a new quantitative method, such as the liquid chromatography with tandem mass spectrometry method to measure the actual amounts of GDF11. Second, the sample size of this study was relatively small. Our results should be verified in another study with larger cohorts to confirm our findings.

\section{Conclusion}

Our findings suggest that physical inactivity was associated with the decreased circulating GDF11, which might be useful for understanding the pathogenesis of COPD. Clarifying the relationships between the physical inactivity and GDF11 may provide a potentially attractive therapeutic approach in COPD via increasing the circulating GDF11.

\section{Acknowledgments}

We thank Dr Shunsuke Ito, Dr Koji Itakura, and Dr Ayumi Mitsune (Tohoku University Graduate School of Medicine, Miyagi, Japan) for recruiting patients and obtaining informed consent. We thank Mr Brent Bell for reading this manuscript. We thank Dr Taku Harada (Tohoku University Graduate School of Medicine, Miyagi, Japan) for help in measuring the quadriceps strength. We thank Dr Satoshi Miyata (Tohoku University Graduate School of Medicine, Miyagi, Japan) for his expert help with statistical analysis.

This study was supported by grants from the Japan Society for the Promotion of Science (grant number: \#16H05307, \#16K15453, \#17H04180) and a grant from the Practical Research Project for Allergic Diseases and Immunology from Japan Agency for Medical Research and Development, AMED (grant number: \#16ek0410018h0002, \#16ek040036h0001, \#17ek0410036h0002).

\section{Author contributions}

RT contributed to the recruiting of patients, obtaining informed consent, biochemical studies, data collection, and interpretation of results. HS designed the study and conducted the interpretation of results, provided technical advice, and writing of the manuscript. MY, AK, NF, SY, TO, and TT (Tsutomu Tamada) provided technical advice and interpretation of results. TI gave technical advice, contributed to interpretation of the results, and writing of the manuscript. KO, TN, KS, YK, HS, SY, MM, and TT (Tsuneyuki Takahashi) contributed to the recruitment of patients and obtaining informed consent. MI designed the study and contributed to the interpretation of results and writing of the manuscript. All authors contributed toward data analysis, drafting and revising the paper and agree to be accountable for all aspects of the work.

\section{Disclosure}

The authors report no conflicts of interest in this work.

\section{References}

1. Barnes PJ. Mechanisms of development of multimorbidity in the elderly. Eur Respir J. 2015;45:790-806.

2. Faner R, Rojas M, Macnee W, Agustí A. Abnormal lung aging in chronic obstructive pulmonary disease and idiopathic pulmonary fibrosis. Am J Respir Crit Care Med. 2012;186:306-313.

3. Mercado N, Ito K, Barnes PJ. Accelerated ageing of the lung in COPD: new concepts. Thorax. 2015;70:482-489.

4. Meiners S, Eickelberg O, Königshoff M. Hallmarks of the ageing lung. Eur Respir J. 2015;45:807-827.

5. Tsuji T, Aoshiba K, Nagai A. Alveolar cell senescence in patients with pulmonary emphysema. Am J Respir Crit Care Med. 2006;174: 886-893.

6. Dagouassat M, Gagliolo JM, Chrusciel S, et al. The cyclooxygenase-2prostaglandin E2 pathway maintains senescence of chronic obstructive pulmonary disease fibroblasts. Am J Respir Crit Care Med. 2013;187: 703-714.

7. Ito K, Barnes PJ. COPD as a disease of accelerated lung aging. Chest. 2009;135:173-180.

8. Tchkonia T, Zhu Y, van Deursen J, Campisi J, Kirkland JL. Cellular senescence and the senescent secretory phenotype: therapeutic opportunities. J Clin Invest. 2013;123:966-972.

9. Nelson G, Wordsworth J, Wang C, et al. A senescent cell bystander effect: senescence-induced senescence. Aging Cell. 2011;11:345-349.

10. Amsellem V, Gary-Bobo G, Marcos E, et al. Telomere dysfunction causes sustained inflammation in chronic obstructive pulmonary disease. Am J Respir Crit Care Med. 2011;184:1358-1366.

11. McPherron AC, Lawler AM, Lee SJ. Regulation of anterior/posterior patterning of the axial skeleton by growth/differentiation factor 11 . Nat Genet. 1999;22:260-264.

12. Loffredo FS, Steinhauser ML, Jay SM, et al. Growth differentiation factor 11 is a circulating factor that reverses age-related cardiac hypertrophy. Cell. 2013;153:828-839.

13. Katsimpardi L, Litterman NK, Schein PA, et al. Vascular and neurogenic rejuvenation of the aging mouse brain by young systemic factors. Science. 2014;344:630-634.

14. Sinha M, Jang YC, Oh J, et al. Restoring systemic GDF11 levels reverses age-related dysfunction in mouse skeletal muscle. Science. 2014;344: 649-652. 
15. Onodera K, Sugiura H, Yamada M, et al. Decrease in an anti-ageing factor, growth differentiation factor 11, in chronic obstructive pulmonary disease. Thorax. 2017;72:893-904.

16. Global initiative for chronic obstructive lung disease. Global strategy for the diagnosis, management, and prevention of chronic obstructive pulmonary disease. 2017 Report. Available from: http://goldcopd.org/. Accessed November 10, 2017.

17. Sugino A, Minakata Y, Kanda M, et al. Validation of a compact motion sensor for the measurement of physical activity in patients with chronic obstructive pulmonary disease. Respiration. 2012;83:300-307.

18. Minakata Y, Sugino A, Kanda M, et al. Reduced level of physical activity in Japanese patients with chronic obstructive pulmonary disease. Respir Investig. 2014;52:41-48.

19. Pitta F, Troosters T, Spruit MA, Probst VS, Decramer M, Gosselink R. Characteristics of physical activities in daily life in chronic obstructive pulmonary disease. Am J Respir Crit Care Med. 2005;171:972-977.

20. Seymour JM, Spruit MA, Hopkinson NS, et al. The prevalence of quadriceps weakness in COPD and the relationship with disease severity. Eur Respir J. 2010;36:81-88.

21. American Thoracic Society Committee on Proficiency Standards for Clinical Pulmonary Function Laboratories. ATS statement: guidelines for the six-minute walk test. Am J Respir Crit Care Med. 2002;166: 111-117.

22. Gosselink R, Troosters T, Decramer M. Peripheral muscle weakness contributes to exercise limitation in COPD. Am J Respir Crit Care Med. 1996;153:976-980.

23. Maltais F, Decramer M, Casaburi R, et al; ATS/ERS Ad Hoc Committee on Limb Muscle Dysfunction in COPD. An official American Thoracic Society/European Respiratory Society statement: update on limb muscle dysfunction in chronic obstructive pulmonary disease. Am J Respir Crit Care Med. 2014;189:e15-e62.
24. Mei W, Xiang G, Li Y, et al. GDF11 protects against endothelial injury and reduces atherosclerotic lesion formation in apolipoprotein E-Null mice. Mol Ther. 2016;24:1926-1938.

25. Shrikrishna D, Patel M, Tanner RJ, et al. Quadriceps wasting and physical inactivity in patients with COPD. Eur Respir J. 2012;40: 1115-1122.

26. Bernard S, LeBlanc P, Whittom F, et al. Peripheral muscle weakness in patients with chronic obstructive pulmonary disease. Am J Respir Crit Care Med. 1998;158:629-634.

27. Watz H, Waschki B, Meyer T, Magnussen H. Physical activity in patients with COPD. Eur Respir J. 2009;33:262-272.

28. Steensberg A, van Hall G, Osada T, et al. Production of interleukin-6 in contracting human skeletal muscles can account for the exerciseinduced increase in plasma interleukin-6. J Physiol. 2000;529 Pt 1: 237-242.

29. Pedersen BK, Febbraio MA. Muscles, exercise and obesity: skeletal muscle as a secretory organ. Nat Rev Endocrinol. 2012;8:457-465.

30. Boström P, Wu J, Jedrychowski MP, et al. A PGC1- $\alpha$-dependent myokine that drives brown-fat-like development of white fat and thermogenesis. Nature. 2012;481:463-468.

31. Ijiri N, Kanazawa H, Asai K, Watanabe T, Hirata K. Irisin, a newly discovered myokine, is a novel biomarker associated with physical activity in patients with chronic obstructive pulmonary disease. Respirology. 2015;20(4):612-617.

32. Moy ML, Teylan M, Weston NA, Gagnon DR, Danilack VA, Garshick E. Daily step count is associated with plasma C-reactive protein and IL-6 in a US cohort with COPD. Chest. 2014;145:542-550.

33. Watz H, Waschki B, Kirsten A, et al. The metabolic syndrome in patients with chronic bronchitis and COPD: frequency and associated consequences for systemic inflammation and physical inactivity. Chest 2009;136:1039-1046. 


\section{Supplementary material}
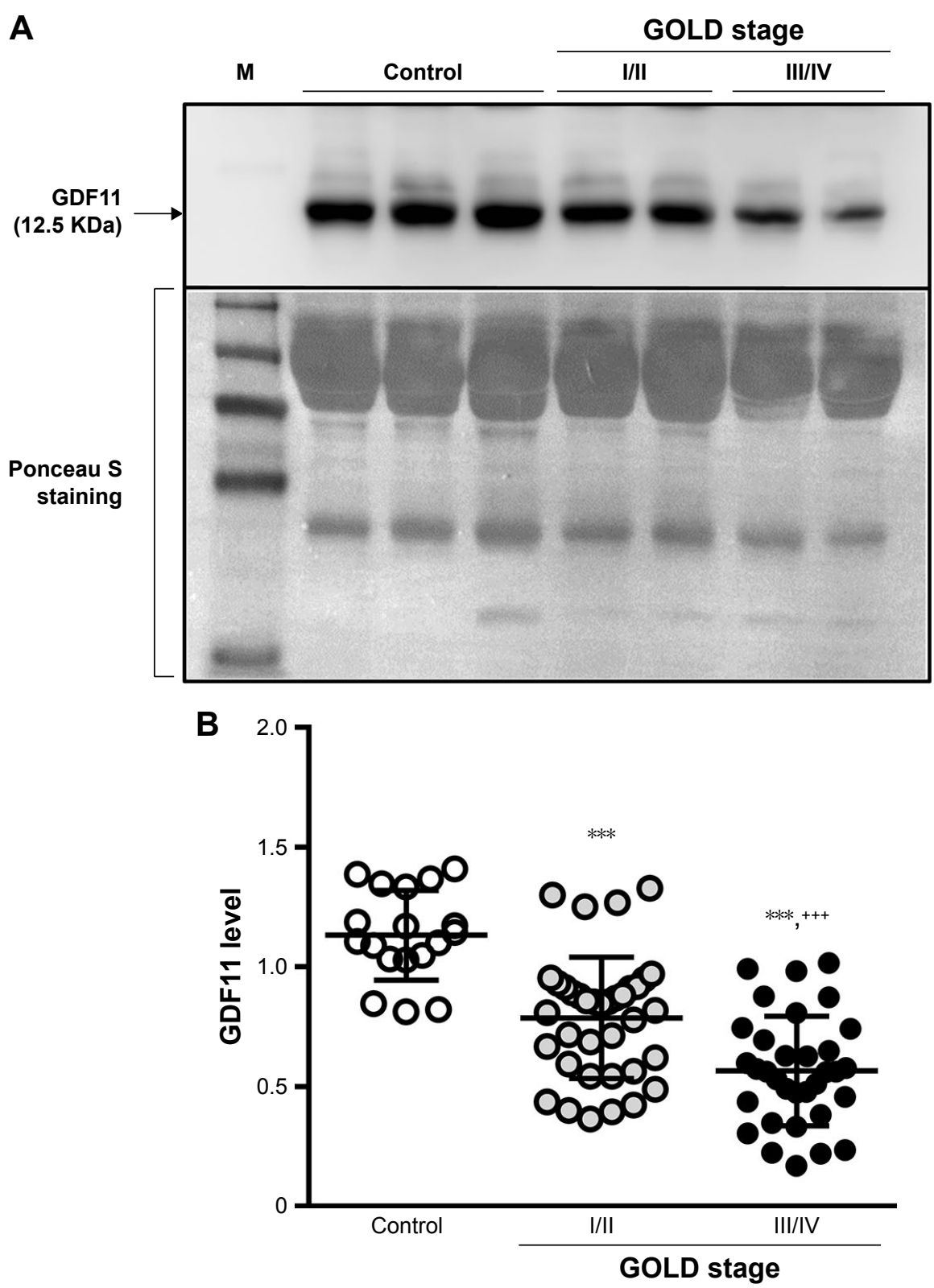

Figure SI Levels of GDFII in plasma.

Notes: Plasma was obtained from the control subjects, GOLD stage I/II and GOLD stage III/IV COPD patients. The levels of plasma GDFII were investigated by immunoblotting (A). Ponceau S staining was used to evaluate the amounts of protein. The GDFII levels were calculated by measuring the intensity of the bands (B). Open circles: control; closed circles (gray): GOLD stage I/II; closed circles (black): GOLD stage III/IV. Results are expressed mean \pm SD. Data were analyzed with one-way ANOVA followed by Tukey's test. *** $p<0.00$ I compared with control; $+++p<0.00$ I compared with GOLD stage I/II.

Abbreviations: ANOVA, analysis of variance; GDFII, growth differentiation factor II; GOLD, Global Initiative for Chronic Obstructive Lung Disease; M, molecular weight marker.

\section{Publish your work in this journal}

The International Journal of COPD is an international, peer-reviewed journal of therapeutics and pharmacology focusing on concise rapid reporting of clinical studies and reviews in COPD. Special focus is given to the pathophysiological processes underlying the disease, intervention programs, patient focused education, and self management protocols.

\section{Dovepress}

This journal is indexed on PubMed Central, MedLine and CAS. The manuscript management system is completely online and includes a very quick and fair peer-review system, which is all easy to use. Visit http://www.dovepress.com/testimonials.php to read real quotes from published authors. 